\title{
Characterization by hyperspectral imaging and hypercolor gamut estimation for structural color prints
}

\author{
Mathieu Hébert ${ }^{1}$, Juan Martínez-García ${ }^{1}$, Thomas Houllier ${ }^{2}$, Hayk Yepremian², Nico- \\ las Crespo-Monteiro ${ }^{1}$, Francis Vocanson ${ }^{1}$, Alain Trémeau ${ }^{1}$, Nathalie Destouches ${ }^{1}$ \\ ${ }^{1}$ Univ Lyon, UJM-Saint-Etienne, CNRS, Institut d Optique Graduate School, Laboratoire Hu- \\ bert Curien UMR 5516, Saint-Etienne, France \\ mathieu.hebert@univ-st-etienne.fr \\ ${ }^{2}$ Institut d'Optique Graduate School, Saint-Etienne, France
}

\begin{abstract}
A recently developed color printing system on glass plates, based on dot-by-dot laser irradiation generating the growth of metallic nanoparticles in a special coating, produces structural colors depending strongly on the illumination and observation configuration. The difficulty for an exhaustive color characterization of the printing technology comes not only from the goniochromaticity of the samples, but also from their very high specularity, to which classical measurement instruments are not adapted. Moreover, as the light-matter interaction relies on a number of optical phenomena (surface plasmon resonance, interferences, diffraction, effects of polarization of light) for which no predictive model is available today, their characterization requires measurement of many printed samples. In this paper, we present a characterization method based on multispectral imaging and on spectral prediction for halftone colors that permitted a first gamut estimation in three specific illumination/viewing configurations.
\end{abstract}

Keywords: Structural colors, Multiview prints, Specular surface, Hyperspectral imaging, Nano-technologies.

\section{Introduction: laser-induced color lusters on glass}

Recent progresses in nanotechnologies enable the coloration of glass with interesting visual rendering. This is for example the case of the technology developed by the laboratoire Hubert Curien, called PICSULP [1], where a coating containing silver [2] is deposited on the glass plate, then irradiated by a laser beam in order to anneal the coating and cluster the metallic ions into metallic nanoparticles (NPs). Goniochromatic coloration of the glass plate surface is thus obtained thanks to various optical phenomena: the presence of silver NPs generates surface plasmon resonance, therefore wavelengthselective absorption as in stained glass [3]; the organization of the NPs along one plane parallel to the coating-air interface generates interferences as in thin films; the NPs can even be aligned along parallel lines, as shown in Figure 1-a, which produces diffraction effects visible at grazing angles, and also gives to the sample a dichroic spectral behavior, i.e. polarization sensitive colors [4,5]. These optical effects are influenced by several physical parameters: the nanoparticle shape, size and spatial organization, as well 
as the coating thickness and refractive index that evolve during the laser treatment. Four command parameters can be varied: exposure (tuned by the laser scanning speed), power, wavelength, and focusing of the laser beam. Polarization of the laser can also be varied.
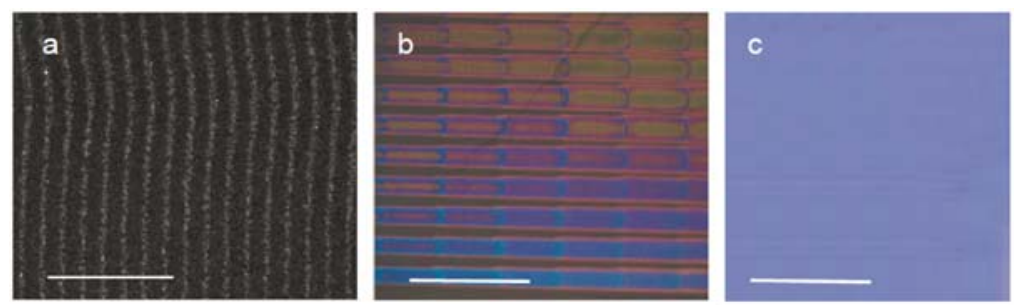

Fig. 1. Printed glass plate observed at (a) nanometric scale where we can see the NPs aligned along parallel lines (scale bar: $2 \mu \mathrm{m}$ ), (b) at microscopic scale where we can see many segments each one printed with different laser parameters (scale bar: $200 \mu \mathrm{m}$ ), and (c) at macroscopic scale in specular reflection mode (scale bar : $2 \mathrm{~mm}$ ).
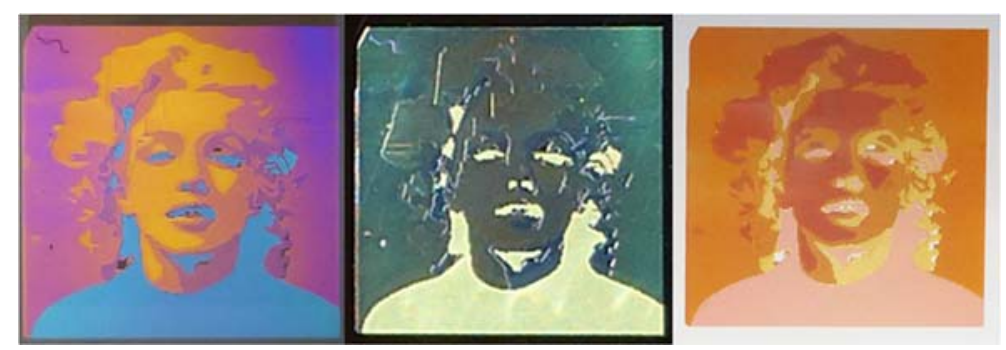

Fig. 2. Color image printed with PICSLUP $(1 \times 1 \mathrm{~cm})$, showing the dramatic color change according to the illumination/observation geometry. Left: Specular reflection mode $\left(45^{\circ}: 45^{\circ}\right.$ geometry); Center: Off-specular reflection mode $\left(45^{\circ}: 0^{\circ}\right.$ geometry); Right: Transmission mode $\left(0^{\circ}: 0^{\circ}\right.$ geometry $)[1]$.

In opposition to classical printing techniques on paper or transparency films where a color patch has "one color" almost independent of the viewing conditions, a glass plate uniformly treated by the PICSLUP technology with constant laser command parameters can display many colors according to the illumination/observation configuration (IOC). Figure 2 shows this dramatic color change through the example of a fourcolor image observed in three IOCs. Therefore, a sample cannot be attributed one color, but should be assigned a set of colors that we propose to call "hypercolor". A second difference with conventional printing is that no model allows predicting the color viewed in a given IOC as a function of the printing command parameters. An exhaustive color characterization therefore requires a huge amount of samples produced each one with different laser command parameters, incrementally varied, to be measured in 
several IOCs. This represents too many measurements for a reasonable calibration process. Moreover, radiances reflected or transmitted in the regular directions (given by Snell's laws) differ from the ones scattered in the rest of the hemisphere by several orders of magnitude: the sensitivity of the measurement setup must therefore be adapted to different IOCs.

In this paper, we present a color characterization method that is not exhaustive, but already representative of the color generation capacities of the PICSLUP technology. It relies on a set of primary colors printed with different laser parameters on one glass plate, and three measurement geometries: specular reflection, off-specular reflection, and normal transmission. Spectral measurements are carried out by using a self-made hyperspectral imaging system. From this set of primary colors, secondary colors can be predicted by juxtaposing lines of different primary colors, and color gamuts can be computed for each of the selected measurement geometries.

\section{Selection of samples and geometries}

In a previous study [6], an exhaustive color characterization has been performed for the $0^{\circ}: 0^{\circ}$ specular reflection mode by measuring the $\mathrm{XYZ}$ color coordinates of the reflected radiances under a A illuminant thanks to a calibrated camera mounted on top of a microscope. This geometry was the only one allowed by the microscope. The colorimetric calibration of the camera was developed in order to suit the very high specularity of the samples by using of a specific color chart presenting as high specularity as the samples. Microscopic patches of 10656 hypercolors, similar to those displayed in Figure 1.b, were printed and analyzed in this way by excluding the edges of each patch. For the present study, we selected a subset of 330 hypercolors printed over $1 \times 1 \mathrm{~mm}$ squares, with the following combinations of laser command parameters [1]: 2 wavelengths ( 488 and $647 \mathrm{~nm}$ ), 3 powers (300, 400, and $600 \mathrm{~mW}$ ), 11 laser scanning speeds $(30000,3200,2000,1400,1200,1000,900,800,600,500$, and $300 \mu \mathrm{m} / \mathrm{s})$ and 5 focus distances $(0,0.5,0.8,1.8$, and $2 \mathrm{~mm})$.

These 330 hypercolors were measured with the hyperspectral imaging setup described in the next section, in three different geometries : $15^{\circ}: 15^{\circ}$ specular reflectance (illumination by collimated light at $15^{\circ}$ from the normal of the sample, and observation in the specular direction), $45^{\circ}: 0^{\circ}$ off-specular reflectance (illumination at $45^{\circ}$ from the normal of the sample, observation along the normal), $0^{\circ}: 0^{\circ}$ transmittance (illumination by collimated light at the normal of the sample, and direct observation of the transmitted collimated beam).

\section{$3 \quad$ Hyperspectral imaging system}

The optical bench assembled for the hyperspectral measurements is shown in Fig. 3. The illumination module was placed on a rotating arm permitting to vary the incidence angle at both sides of the sample; it comprised an optical fiber connected to a light source (HL-2000-HP 20W halogen lamp by Ocean Optics) placed at the focus of a lens 
in order to obtain a collimated light beam of $4 \mathrm{~cm}$ diameter. The detection module comprised a lens-free 12-bit gray-level camera, a set of 31 interferential filters of $10 \mathrm{~nm}$ spectral width centered every $10 \mathrm{~nm}$ from 400 to $700 \mathrm{~nm}$, and a telecentric lens made of two lenses whose foci meet each other, the sample being placed in the other focal plane of the first lens, the detector of the camera in the one of the second lens [7]. The advantage of the telecentric lens is that it allows the simultaneous measurement of many patches, in strictly similar measurement conditions. This is a crucial point for our very specular samples since a very small angular variation may modify considerably the detected radiance (variations up to $50 \%$ can be easily observed, which is still small in comparison to the $10^{10}$ dynamic of the BRDF of the samples). In this context, the telecentric lens is crucial to ensure that all points of the sample are viewed under the same angle. With a conventional lens, the image of a uniform hypercolor patch would be non-uniform because of the goniochromaticity of the sample and the oblicuity of the captured rays at the border of the image.

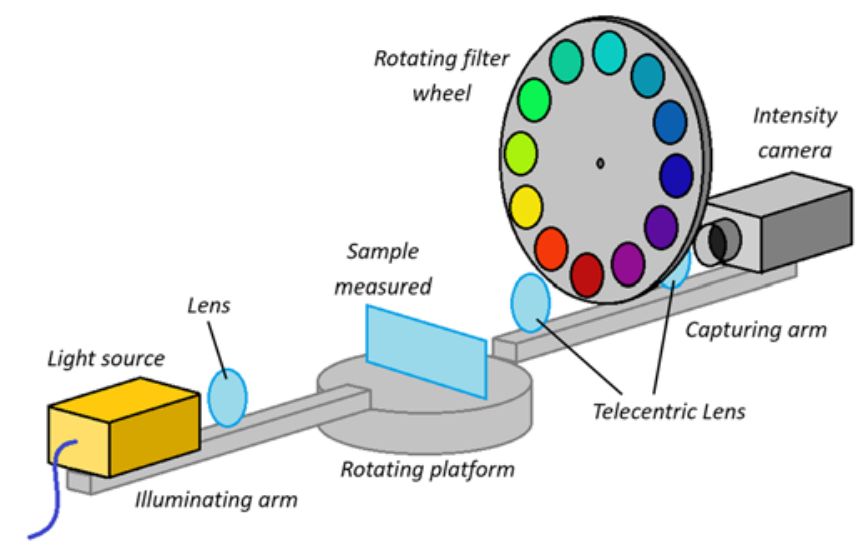

Fig. 3. Scheme of the hyperspectral imaging system for the measurement of samples, here in transmission mode.

The calibration of the spectral bench was done by using a neutral color reference sample reflecting or transmitting similar radiance as the studied samples. In specular reflection mode, the reference sample was a silicon mirror whose spectral reflectance was measured with a spectrophotometer in the $15^{\circ}: 15^{\circ}$ geometry. Once placed in the hyperspectral bench, for each band-pass filter (denoted by its central wavelength $\lambda$ ), the aperture and exposure of the camera was adjusted in order to obtain a maximum signal without saturation of the camera. These camera parameters as well as the captured images $I_{\text {ref }}(x, y, \lambda)$ of the reference sample were stored, then the images $I(x, y, \lambda)$ of the PICLSUP print were captured by using for each band-pass filter the same camera parameters as those used for the reference sample. Lastly, we considered for each pixel $(x, y)$ and each waveband $\lambda$, the ratio $I(x, y, \lambda) / I_{r e f}(x, y, \lambda)$, which corresponds to a reflectance factor. 
In off-specular reflection mode, the reference sample was a green diffusing ceramic tile, whose spectral reflectance was comparable in all wavebands to the one of the samples (these latter exhibiting a greenish hue in this IOC). Since the BRDF of our specular samples is extremely low at $45^{\circ}$ from the illumination direction, we illuminated them with a high conical irradiance coming from an integrating sphere with a $500 \mathrm{~lm}$ halogen lamp in it. Similar procedure as for the specular reflection mode was then applied.

In transmittance mode, the reference sample was simply void. Once again, similar procedure as for the specular reflection mode was applied. By way of illustration, we show in Figure 4 the images of the sample and the reference sample captured in two wavebands around $460 \mathrm{~nm}$ and $600 \mathrm{~nm}$. Note that spatial variations of the illuminance, lens transmittances, vigneting and other aberrations introduced by the system, are systematically corrected as they are similar in both the sample and reference images.

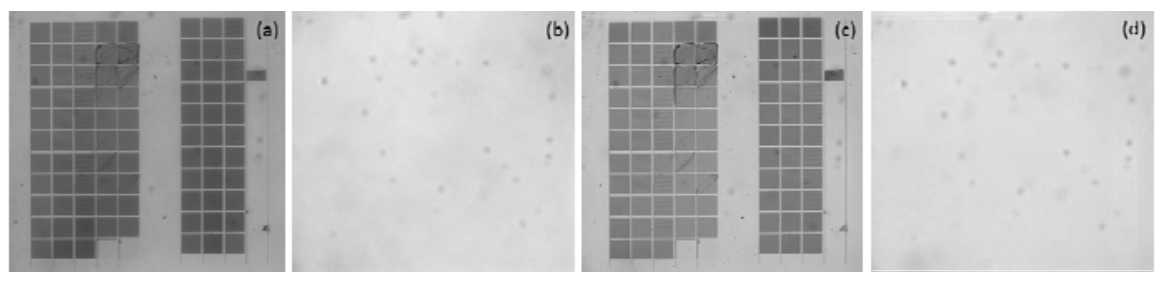

Fig. 4. (a, c) Images of a sample printed by the PICSLUP system and (b, d) images of the reference sample (void) (b,d) obtained in transmission mode at $460 \mathrm{~nm}(\mathrm{a}, \mathrm{b})$, and $600 \mathrm{~nm}(\mathrm{c}, \mathrm{d})$.

From the 31 pairs of images obtained in each mode, we can reconstruct the hyperspectral image with a wavelength sampling of $10 \mathrm{~nm}$, which is a rather good spectral $(10 \mathrm{~nm})$ and spatial $(1024 \times 768$ pixels $)$ resolution compared to hyperspectral imaging systems with variable lighting geometry existing today $[8,9]$. Before obtaining the hyperspectral image, an important step is the registration of the different images because the band-pass filters can introduce a small shift between the different images captured [10]. An image processing applet was developed to perform registration automatically.

At the end of the process, we have the spectral and spatial information of the prints. A good way to visualize their colors in a computer display is to render them by assuming an illuminant to obtain their CIE-XYZ values and then obtain SRGB colors by using standard transformations. In Figure 5, we can see a color version of the hyperspectral images obtained in the three measurement geometries, by assuming a D65 illuminant. We can notice the dramatic change in color of the same printed areas according to the IOC used. Some patches look scratched in the specular reflectance mode: a surface pealing effect due to thermal and mechanical strains produced by the laser treatment in specific parameter ranges can roughen the surface, which thus reflects a much lower radiance in the specular direction. 

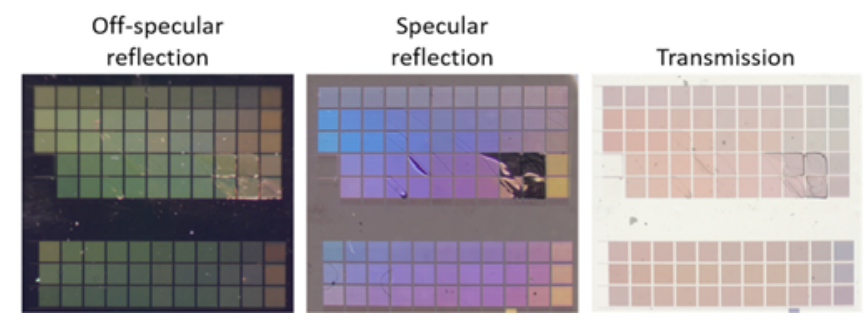

Fig. 5: sRGB color images rendered from the hyperspectral images in three different measurement geometries. The areas of the squares are: $1 \mathrm{~mm} \times 1 \mathrm{~mm}$.

Each hyperspectral image obtained contains one spectrum per pixel. By averaging the spectra of pixels belonging to a same patch, we could obtain the spectra of all the printed patches and calculate the corresponding CIE 1976 LAB color coordinates by assuming a D65 illuminant (Figure 6). In off-specular reflection mode, the volume of colors that can be obtained have a moderate size, with a strong presence in the green hue which are lacking in the other configurations. The specular reflection mode produces a wider range of chromaticities in the samples. However, the variation in lightness in that geometry is rather limited, presenting L* values between 40 and 65 units. The colors in the back side of the samples in this configuration have a much smaller volume, with very reduced chromatic and lightness variations. In transmission mode, the color gamut is very reduced, consisting mainly of very light and pale colors of orange hue.
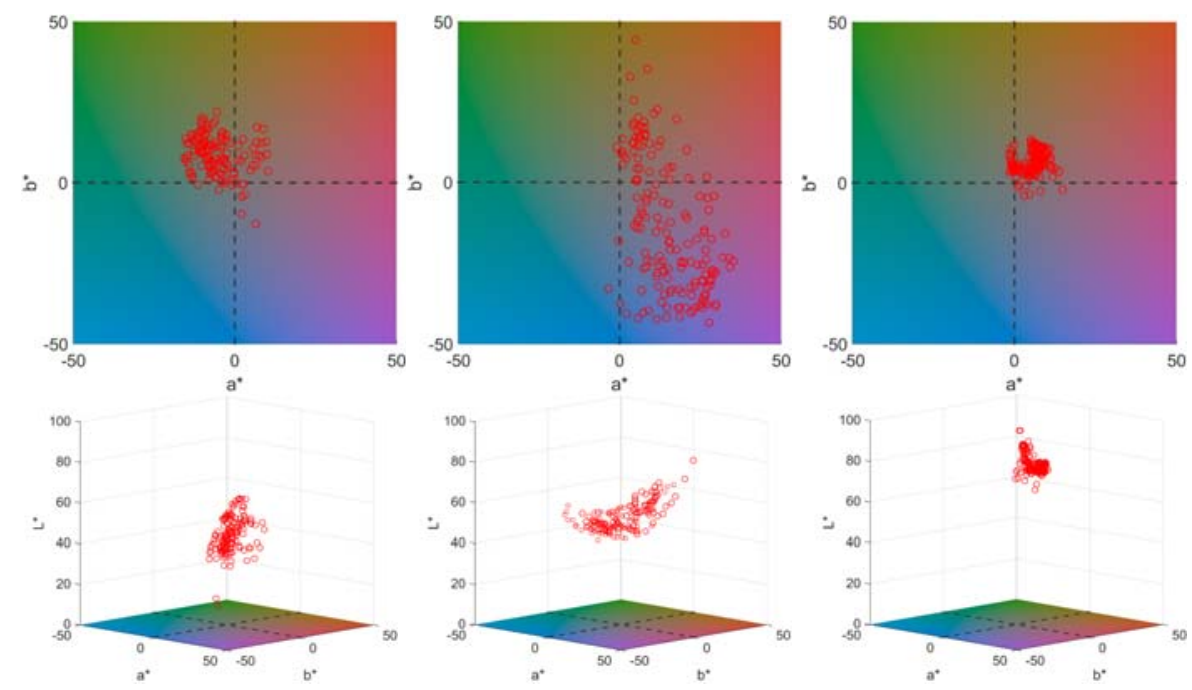

Fig. 6. Colors obtained for the $45^{\circ}: 0^{\circ}$ off-specular reflection geometry (left), $15^{\circ}: 15^{\circ}$ specular reflection geometry (center) and $0^{\circ}: 0^{\circ}$ transmission geometry (right) of the 330 printed hypercolor patches, represented in the CIE $1976 \mathrm{~L}^{*} \mathrm{a} * \mathrm{~b} *$ color space (bottom line) and projected onto the $\left(\mathrm{a}^{*}, \mathrm{~b}^{*}\right)$-plane (top line). 


\section{$4 \quad$ Gamut extension by halftoning}

In contrast with ink-based printers which need halftoning in order to reproduce many colors from their limited set of inks (four inks in quadrichromy), the PICSLUP technology can produce a wide variety of hypercolors in each point, and cover the plate with one uniform hypercolor (continuous tone printing). However, since only 330 hypercolors have been characterized, we propose to use halftoning to obtain additional, intermediate hypercolors. Since the set of printable halftones is considerable, we wanted to know whether it is possible to predict their color in the OIC instead of measuring them. We thus propose to verify the validity of a classical prediction model for halftone colors in case of low light scattering in the printing support: the Spectral Neugebauer model [11].

A set of 30 different $2 \times 2 \mathrm{~mm}$ patches where printed on the same glass support, with different combinations of five primaries and different proportions of area coverage; line halftoning was used by juxtaposing lines of $20 \mu \mathrm{m}$ width. An image of the squares, captured with the hyperspectral imaging system in the $15^{\circ}: 15^{\circ}$ specular reflection configuration is shown in Figure 7, as well as the composition of each of the printed patches. The leftmost column corresponds to patches wholly covered by one primary. These are used as inputs in the prediction models in order to estimate the colors obtained from the combination of several primaries. The remaining 25 patches correspond to areas printed with the combinations of two or three different primaries. The primaries used are specified by the numbers in the brackets, and the ratio of the area covered by each of them is specified below the primaries. For example, the square located in the $2^{\text {nd }}$ row and $5^{\text {th }}$ column is made with the primaries 1 and 5 , with a ratio of 2 lines of Primary 1 for each line of Primary 5.

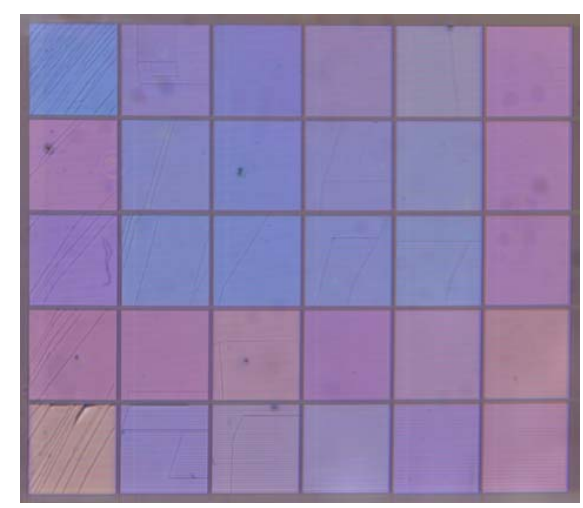

\begin{tabular}{|c|c|c|c|c|c|}
$\begin{array}{c}\text { Full } \\
\text { Primary 1 }\end{array}$ & $\begin{array}{c}(1,2) \\
1: 1\end{array}$ & $\begin{array}{c}(1,3) \\
1: 1\end{array}$ & $\begin{array}{c}(1,4) \\
1: 1\end{array}$ & $\begin{array}{c}(1,5) \\
1: 1\end{array}$ & $\begin{array}{c}(2,3) \\
1: 1\end{array}$ \\
\hline $\begin{array}{c}\text { Full } \\
\text { Primary 2 }\end{array}$ & $\begin{array}{c}(1,2) \\
2: 1\end{array}$ & $\begin{array}{c}(1,3) \\
2: 1\end{array}$ & $\begin{array}{c}(1,4) \\
2: 1\end{array}$ & $\begin{array}{c}(1,5) \\
2: 1\end{array}$ & $\begin{array}{c}(2,3) \\
2: 1\end{array}$ \\
\hline $\begin{array}{c}\text { Full } \\
\text { Primary 3 }\end{array}$ & $\begin{array}{c}(1,2) \\
3: 1\end{array}$ & $\begin{array}{c}(1,3) \\
3: 1\end{array}$ & $\begin{array}{c}(1,4) \\
3: 1\end{array}$ & $\begin{array}{c}(1,5) \\
3: 1\end{array}$ & $\begin{array}{c}(2,3) \\
3: 1\end{array}$ \\
\hline $\begin{array}{c}\text { Full } \\
\text { Primary 4 }\end{array}$ & $\begin{array}{c}(2,4) \\
1: 1\end{array}$ & $\begin{array}{c}(2,5) \\
1: 1\end{array}$ & $\begin{array}{c}(3,4) \\
1: 1\end{array}$ & $\begin{array}{c}(3,5) \\
1: 1\end{array}$ & $\begin{array}{c}(4,5) \\
1: 1\end{array}$ \\
\hline Full & $(1,2,3)$ & $(1,2,5)$ & $(1,3,5)$ & $(2,1,3)$ & $(2,4,5)$ \\
\hline Primary 5 & $1: 1: 1$ & $1: 1: 1$ & $1: 1: 1$ & $2: 1: 1$ & $2: 1: 1$ \\
\hline
\end{tabular}

Fig. 7. Image of the squares printed for halftoning prediction testing, captured with the hyperspectral imaging bench. Specification of squares printed for halftoning evaluation. The squares on the leftmost column were printed by using only one primary, while the other 25 were printed by combining the primaries in the parenthesis in the proportions shown. 

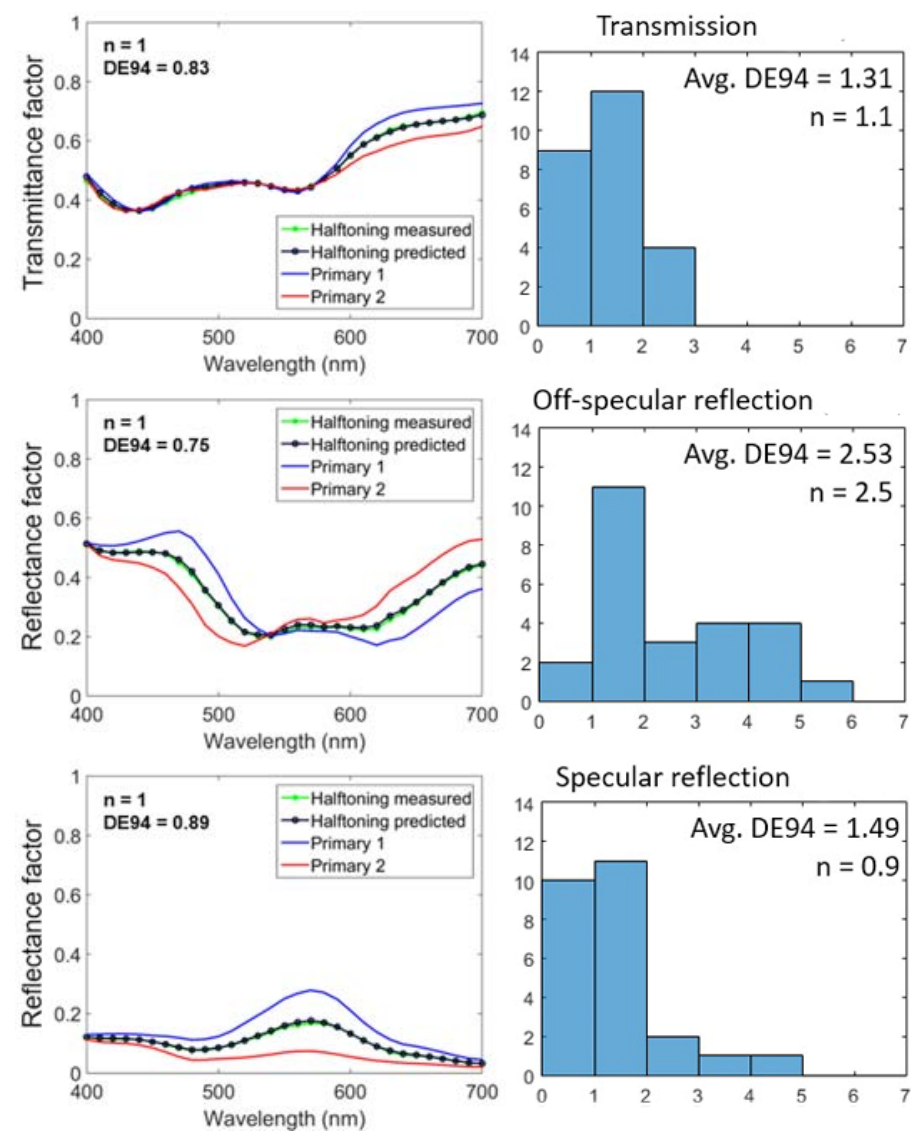

Fig. 8. Left: Measured and predicted spectra of one halftone patch and measured spectra of its composing primaries in the three measurement modes: transmission (top row), specular reflection (middle row), off-specular reflection (bottom row). Right: histograms of the DE94 color

distances between the measured and predicted spectra for the 20 halftone samples tested.

The spectral properties of the samples were measured in the three IOCs presented in Section 3. The Spectral Neugebauer model predicts, for a given IOC, the spectrum of a halftone hypercolor, $S_{\text {halfone }}(\lambda)$, as a function of the spectra $S_{i}(\lambda)$ of the primaries $i$ and their respective surface coverage $a_{i}$, as:

$$
S_{\text {halfone }}(\lambda)=\sum_{i} a_{i} S_{i}^{1 / n}(\lambda)
$$

Figure 8 shows the measured and predicted spectra of the first halftone patch, located in the first row and second column of the sample (see Figure 7), for each of the different measurement configurations. The prediction accuracy, assessed by the CIE94 color difference between the measured and predicted colors by assuming a D65 illuminant, is rather good, considering that the just-noticeable-difference threshold of the human visual system is approximately 1 unit. The prediction accuracy for the Neugebauer model 
over the set of samples that we produced is slightly poorer than the one usually achieved in color reproduction by digital printers (e.g. inkjet on photo-quality paper), e.g. the Yule-Nielsen modified Spectral Neugebauer model which is one of the more efficient [12]. In the present case, as expected since our samples are non-scattering, no improvement is achieved by using the Yule-Nielsen modified Spectral Neugebauer model. The PICSLUP printing system is probably less reproducible than commercial digital printers, especially due to small artifacts which may have small but noticeable impact on the spectral properties of the prints. Moreover, the measurement process is also subject to some imprecision and noise. Being given these constraints, we can be satisfied with the prediction accuracy that is achieved.

By using the Spectral Neugebauer model, we can predict the colors that can be produced in each geometrical configuration by the combination of the numerous primaries produced by the PICSLUP system, shown in Fig. 6. To obtain the complete set of colors that can be produced with the PICSLUP system, we exhaustively predicted the colors produced by the halftoning mixture of every pair of primaries from the set of 330 hypercolor patches. We found that in all the IOCs, the predicted colors obtained by halftoning with each pair of primaries draw an almost straight line between them, as a consequence of the linear equation (1). This volume of colors is very close to the convex hull of the set of primaries used, i.e., the minimum convex volume that contains all the primaries in a given configuration. The color gamut in each of the geometries can therefore be approximated in a simple manner as the convex hull defined by the primaries used. The extended color gamuts obtained for each of the six measurement configurations thanks to use of halftoning are presented in Figure 9.
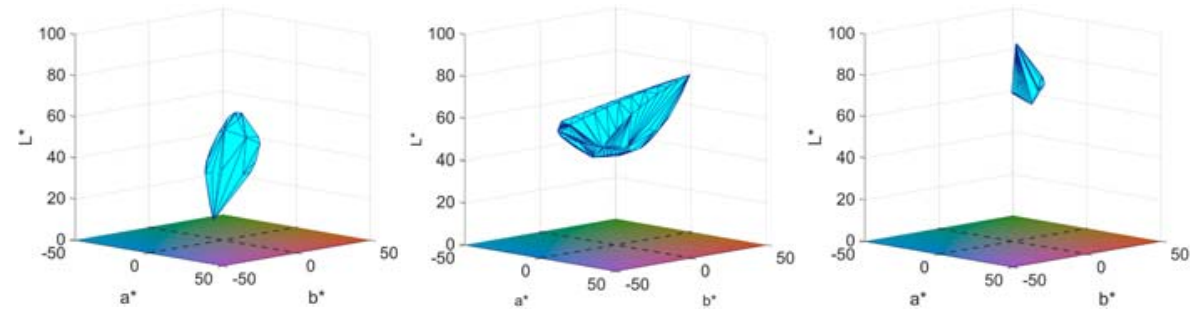

Fig. 9. Estimated color gamut volumes for the PICSLUP technology in the CIE 1976 L*a*b* color space for the $45^{\circ}: 0^{\circ}$ off-specular reflection geometry (left), $15^{\circ}: 15^{\circ}$ specular reflection geometry (center) and $0^{\circ}: 0^{\circ}$ transmission geometry (right).

Despite the photometric constraints and limitations that exist for characterizing the PICSLUP technology, we demonstrated that line halftoning was possible in our system by obtaining a satisfactory accuracy in the prediction of the colors that can be produced by mixing different primaries. The worst accuracy was obtained in the off-specular reflection mode where the reflected radiance was very low, which made the measurements prone to errors due to stray light or diffraction effects. We can see by comparing Figures 6 and 9 that the volume of producible colors notably increased and became more convex and continuous. This is important for color imaging, where a continuous sets of colors are necessary to produce gradients present in real images. 


\section{$5 \quad$ Multiview imaging}

Another advantage introduced by halftoning is that the amount of hypercolors is considerably increased, displaying each one a different set of colors in the different IOCs. It is then easier to obtain parameters to print areas that visually match a given color $A$ in one geometry while matching another target color B in a different geometry. This opens the possibility for some interesting imaging design and security applications. For example, we can hide patterns in a background by selecting different sets of command parameters for the patterns and the background that display the same color in one geometry and reveal different colors in another geometry.

By way of illustration, we designed a 2-geometry pattern from samples measured by our hyperspectral imaging system. Figure 10 shows the colors produced in two different geometrical configurations by the four sets of parameters selected. These colors are then used to design the spatial arrangement of the areas that will be printed with each of the selected parameter sets. Figure 11 shows the spatial arrangement we designed to produce our 2-geometry pattern, as well as the resulting colors produced displayed in the two geometries.
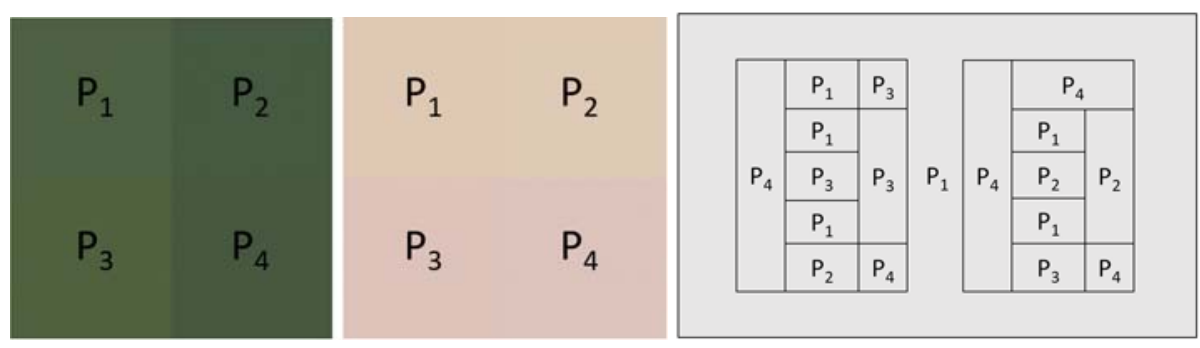

Fig. 10. Left and middle: Rendered sRGB color of four different hypercolors $\mathrm{P} 1, \mathrm{P} 2, \mathrm{P} 3$ and $\mathrm{P} 4$, in the $45^{\circ}: 0^{\circ}$ off-specular reflection (left) and $0^{\circ}: 0^{\circ}$ transmission (middle) geometries. Right: Spatial arrangement designed with the four hypercolors in order to produce the 2-geometry pattern displayed in Figure 11.
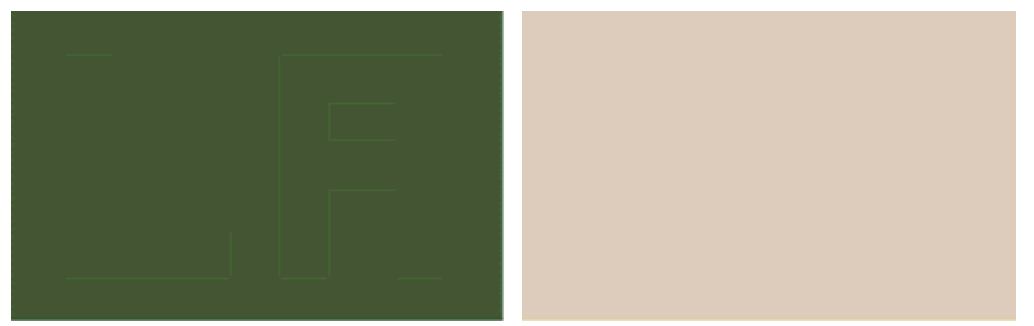

Fig. 11. Rendered sRGB colors of a 2-geometry pattern observed in the $45^{\circ}: 0^{\circ}$ off-specular reflection (left) and $0^{\circ}: 0^{\circ}$ transmission (middle) geometries. 
This approach can be extended to multicolor images, and it is probable that more than two different images can be displayed. The strong color variations that polarizing filters can generate is also a way of increasing the number of possible configurations [5]. However, multi-gamut mapping would be necessary to automatically determine the hypercolors, if any, that would be able to display the different targeted images, and no multi-gamut mapping technique is available today: a challenge for the incoming years.

\section{Conclusions}

In the paper, we presented a spectral and color characterization of glass plates coated with a layer containing composite layer nanostructured by laser irradiation, which exhibits different "hypercolors" (i.e., sets of colors displayed in different illumination/observation configurations) in each point. Despite the difficulty of characterizing the color appearance of these strongly goniochromatic, transparent and specular samples, we could find a suitable measurement method thanks to hyperspectral imaging, where the camera is preceded by a telecentric lens. Several patches can thus be simultaneously measured, while being all viewed under the same angle independently of their position in the sample, thereby in the image. Special attention must be paid to the choice of the reference sample, which should have similar reflectance or transmittance as the studied sample in the considered measurement geometry in order to avoid a too low or excessive light signal detected. We then showed that, once knowing the spectral reflectance or transmittance of pure hypercolors (primaries), we can estimate, with rather good accuracy in this context, the spectral properties of halftone hypercolors obtained by juxtaposing thin lines of different primaries. This finally enables estimating to the color gamut of the printing technology in the considered illumination/observation geometry, and opens interesting perspectives for the production of multi-view still images.

\section{Acknowledgement}

This work was supported by the French National Research Agency (ANR) within the program "Investissements d'Avenir" (ANR-11-IDEX-0007), in the framework of project PHOTOFLEX ${ }^{\circ}$ ANR-12-NANO-0006 and the LABEX MANUTECH-SISE (ANR-10-LABX-0075) of Université de Lyon.

\section{References}

1. J. Martinez, M. Hébert, A. Trémeau, N. Destouches, "Multi-color properties of silver glaze images photo-engraved on glass plates," Color Imaging Conference (Darmstadt, Germany, 19-23 October 2015).

2. F. Tricot, F. Vocanson, D. Chaussy, D. Beneventi, S. Reynaud, Y. Lefkir, and N. Destouches, "Flexible photochromic TiO2:Ag thin film elaborated by printing techniques." in E-MRS 2015, Spring Meeting, symposium H, Nanoparticles in dielectric matrix for electronics and optics: from the fabrication to the devices, 2015. 
3. National Research Council, Controlling the Quantum World: The Science of Atoms, Molecules, and Photons. Chapter 6, "Photonics and the nanoworld," Washington, DC: The National Academies Press, 2007.

4. N. Destouches, J. Martínez-García, M. Hébert, N. Crespo-Monteiro, G. Vitrant, Z. Liu, A. Trémeau, F. Vocanson, F. Pigeon, S. Reynaud, Y. Lefkir, "Dichroic colored luster of laserinduced silver nanoparticle gratings buried in dense inorganic films," J. Opt. Soc. Am. B 31, C1-C7 (2014).

5. N. Destouches, N. Crespo-Monteiro, T. Epicier, Y. Lefkir, F. Vocanson, S. Reynaud, R. Charrière, M. Hébert, "Permanent dichroic coloring of surfaces by laser-induced formation of chain-like self-organized silver nanoparticles within crystalline titania films," Conf. Synthesis and Photonics of Nanoscale Materials X, Proc. of SPIE Vol. 8609-860905 (2013).

6. J. Martinez, M. Hébert, A. Trémeau, "Color calibration of an RGB digital camera for the microscopic observation of highly specular materials," Proc. SPIE 9398, SPIE/IS\&T Electronic Imaging Symposium, Measuring, modeling, reproduction materials appearance (San Francisco, USA, 8-12 February 2015).

7. M. Lequime, M. Zerrad, C. Deumié, C. Amra, "A goniometric light scattering instrument with high-resolution imaging," Optics Communications 282, 1265-1273 (2009).

8. P. Seroul, M. Hébert, and M. Jomier, "Hyperspectral imaging system for in-vivo quantification of skin pigments," in IFSCC, pp. 123-132, 2014.

9. R. Jolivot, Y. Benezeth, and F. Marzani, "Skin parameter map retrieval from a dedicated multispectral imaging system applied to dermatology/cosmetology," Journal of Biomedical Imaging, vol. 2013, p. 26, 2013.

10. C. Zhang, M. Rosenberger, A. Breitbarth, G. Notni, "A Novel 3D Multispectral Vision System based on Filter Wheel Cameras," Proceedings of IEEE International Conference on Imaging Systems and Techniques (IST), Chania, Greece, October 4-6 2016.

11. Raja Balasubramanian, A spectral Neugebauer model for dot-on-dot printers, Proc. SPIE vol. 2413, (1995).

12. M. Hébert and R. Hersch, "Review of spectral reflectance models for halftone prints: Principles, Calibration, and prediction accuracy," Color Res. Appl., vol. 40, no. 4, pp. 383-397, 2015. 